\title{
On the spontaneous stochastic dynamics of a single gene: complexity of the molecular interplay at the promoter
}

Antoine Coulon ${ }^{1,2,3^{*}}$, Olivier Gandrillon ${ }^{1,3}$, Guillaume Beslon ${ }^{2,3}$

\begin{abstract}
Background: Gene promoters can be in various epigenetic states and undergo interactions with many molecules in a highly transient, probabilistic and combinatorial way, resulting in a complex global dynamics as observed experimentally. However, models of stochastic gene expression commonly consider promoter activity as a twostate on/off system. We consider here a model of single-gene stochastic expression that can represent arbitrary prokaryotic or eukaryotic promoters, based on the combinatorial interplay between molecules and epigenetic factors, including energy-dependent remodeling and enzymatic activities.

Results: We show that, considering the mere molecular interplay at the promoter, a single-gene can demonstrate an elaborate spontaneous stochastic activity (eg. multi-periodic multi-relaxation dynamics), similar to what is known to occur at the gene-network level. Characterizing this generic model with indicators of dynamic and steady-state properties (including power spectra and distributions), we reveal the potential activity of any promoter and its influence on gene expression. In particular, we can reproduce, based on biologically relevant mechanisms, the strongly periodic patterns of promoter occupancy by transcription factors (TF) and chromatin remodeling as observed experimentally on eukaryotic promoters. Moreover, we link several of its characteristics to properties of the underlying biochemical system. The model can also be used to identify behaviors of interest (eg. stochasticity induced by high TF concentration) on minimal systems and to test their relevance in larger and more realistic systems. We finally show that TF concentrations can regulate many aspects of the stochastic activity with a considerable flexibility and complexity.

Conclusions: This tight promoter-mediated control of stochasticity may constitute a powerful asset for the cell. Remarkably, a strongly periodic activity that demonstrates a complex TF concentration-dependent control is obtained when molecular interactions have typical characteristics observed on eukaryotic promoters (high mobility, functional redundancy, many alternate states/pathways). We also show that this regime results in a direct and indirect energetic cost. Finally, this model can constitute a framework for unifying various experimental approaches. Collectively, our results show that a gene - the basic building block of complex regulatory networks - can itself demonstrate a significantly complex behavior.
\end{abstract}

\section{Background}

Considered for a long time to be insignificant variations around a significant mean, stochasticity in gene expression is now clearly demonstrated to be important in many situations and in many organisms [1-16] and to participate in various biological processes [15-20], as formerly proposed [21]. The molecular bases of this

\footnotetext{
* Correspondence: antoine.coulon@cgmc.univ-lyon1.fr

'Université de Lyon, Université Lyon 1, Centre de Génétique Moléculaire et Cellulaire (CGMC), CNRS UMR5534, F-69622 Lyon, France
}

stochasticity are multiple and constitute now a major subject of investigation. They are frequently distinguished between intrinsic and extrinsic stochasticity [1,22]. Although this distinction requires a clear statement of the considered system [23], this system is often (eg. as in [1]) implicit and corresponds to what we would call a "node" in a regulatory network. Then, extrinsic and intrinsic stochasticity are respectively the propagation through this node of global fluctuations of the concentration of transcription factors (TFs), RNA polymerase ...,
C Biomed Central

C 2010 Coulon et al; licensee BioMed Central Ltd. This is an Open Access article distributed under the terms of the Creative Commons Attribution License (http://creativecommons.org/licenses/by/2.0), which permits unrestricted use, distribution, and reproduction in any medium, provided the original work is properly cited. 
and the generation of randomness due to the molecular events, discrete and probabilistic in nature, that take place within the system (TFs binding to the promoter, transcription initiation, RNA degradation ...).

A major challenge in this field is to isolate and characterize the various sources of stochasticity in different organisms (from prokaryotes to higher eukaryotes) by theoretical [22-44] and/or experimental means [1-12]. Stochasticity gets significant when some discrete molecular events become rare. Typically, when RNA or proteins are in low copy number, synthesis and degradation events are rare and represent important variations relatively to the total amount of these molecular species. This generates a so-called Poisson noise at both RNA and protein levels [23]. Also, because at low copy number TFs cannot be considered to be uniformly distributed, spatial proximity of the few TFs to the promoter becomes important and contributes to enhance stochasticity [33]. Finally, and independently of molecule concentrations, the transition of the promoter between different states (chromatin state, presence/absence of a TF ...) provokes heterogeneity in transcription (eg. bursts $[3,4,6,14,15])$ and appears to be a major source of stochasticity [5].

The vast literature describing the molecular machinery involved at the promoter reports very elaborate properties. The various classes of molecules involved in transcriptional regulation show quite wide-ranging but surprisingly short residence time (typically few seconds) within complexes [45-48]. Moreover, despite this rapid turnover of most (if not all) molecules, eukaryotic regulatory complexes also demonstrate a clear slow-timescale activity: They proceed through a periodic pattern of occupancy level by molecules, conformal changes and epigenetic modifications with a precise timing and a period in the order of few tens of minutes [48-52]. This phenomenon referred to as the cyclical recruitment, occupancy pattern or loading profile of molecules on the promoter, or even as chromatin breathing. This provides a new vision of regulatory complexes as highly dynamic structures in constant assembly and disassembly, with alternative functionally redundant pathways of formation and with phenomena occurring concomitantly at different timescales [48,52-55]. An important point is that, in both prokaryotes [56,57] and eukaryotes [58-61], the association/dissociation of most molecules involves cooperation and competition with the other molecules bound to the promoter. Also, alternative conformations (DNA looping, chromatin open/closed state, nucleosome position along DNA ...), post-translational covalent modifications of histone tails residues (acetylation, methylation, phosphorylation ... defining the "histone code" [62]) and DNA methylation are other factors that influence and are influenced by the molecules present on the promoter in a dynamic, highly combinatorial and possibly energy- dependent manner [53,60-66]. These combinatorial aspects also take place in RNA polymerase recruitment $[49,50,66]$ and provides the promoter with a variety of levels of transcriptional competency $[48,56,61,65]$, far from the binary vision of all-or-nothing active/inactive genes. All this elaborate molecular interplay provides the regulatory structure with a complex dynamics and certainly have major outcomes on stochasticity of gene expression. Two-state on/off promoters with exponential switching times $[3,4,14,23-27,30,32,34-37,40]$ (or slightly more detailed models $[2,33,44]$ ) have been used for a long time and gave many valuable insights into the importance of promoter dynamics. Beyond this simple description, few authors recently focused on more precise descriptions of promoters from the viewpoint of stochastic gene expression $[41-43,67,68]$. These studies revealed interesting results but remained focused on specific features (eg. non-exponential waiting times, shape of regulatory input function) or on restricted systems (eg. prokaryotic energetically-closed systems) so that general principles on the capacity of regulatory molecular interplay remain mostly unexplored. Recently, the stochastic dynamics of a eukaryotic promoter has been modeled considering the interplay between TFs and chromatin modifications and in relation with experimental data [52]. This work provided several important insights into the relation between single-cell and population dynamics and showed that the approach is very promising. However, being essentially based on simulations, the understanding of the structure of the dynamics and all its potentiality as well as parameter explorations remain more limited than when using an analytical approach. Moreover, the very synthetic metrics commonly used in studies of stochastic gene expression (eg. variance normalized by square mean) miss most of both steady-state and dynamic aspects of the system's activity. Here, we employ more comprehensive measures such as power spectra or autocorrelations $[7,8,12,29,30,33,34,69,70]$ and full distributions $[2,6,9,27,28,35-37,40]$ that are known to reveal many more relevant features. A power spectrum is a measure that describes the temporal fluctuations of a signal (eg. a protein level) by revealing its frequency content. For instance, a peak in a power spectrum at a given frequency tells that the considered signal tends to repeat itself periodically (the sharper the peak, the more precisely the signal is repeated). On the opposite, a plateau up to a given frequency followed by a decrease tells that the signal fluctuates aperiodically with a typical correlation time as short as the plateau spans over high frequencies.

We first present a generic promoter-centered model of the stochastic expression of a single gene - that can represent arbitrary regulatory systems from prokaryotes to eukaryotes - and describe its spontaneous activity in terms of power spectra, normalized variance and full 
distribution. It reveals that a single gene can demonstrate the same type of complex dynamics as those that were previously identified at the network-level (eg. multi-periodic multi-relaxation dynamics). In particular, it can reproduce with realistic parameters the periodic occupancy patterns observed experimentally on eukaryotic promoters and highlight the central role of energy-dependence in this context. Then we show that instantiation into minimalist systems can help to identify novel properties (eg. stochasticity induced at high TF concentration) and verify their applicability to larger and more biologically plausible systems. Finally, we show how TF concentration can modulate many aspects of the promoter activity in a highly complex and flexible manner, suggesting the transcriptional regulation as a central piece for the cell to control and take advantage of stochasticity. We discuss our results and their theoretical and experimental implications in different fields.

\section{Methods}

Dedicated to the study of the impact of stochastic promoter dynamics on gene expression, this model (figures $1 \mathrm{~A}$ and 1C) describes the molecular events of various sorts occurring at the promoter. Mechanisms subsequent to transcription initiation are kept simple (as in most models) but explicit so that we can assess how promoter stochasticity propagates up to RNA and protein levels and confronts to other sources of gene-intrinsic stochasticity. The reader can refer to the Additional file 1 for an extensive description of the model. For simplicity, we only give here a brief description of a simplified version. However, all derivation and conclusions of this paper stand for both versions. The following model shares some similarities with previous models $[42,52,67,68,71-73]$ and can be solved as an instance of generic techniques $[23,34,69,70,74,75]$ or simulated using generic frameworks [76-78]. We highlight here the key difference in its definition, interpretation and resolution.

Molecular interplay at the promoter - Kinetic formulation We first consider TF molecules associating with and dissociating from the promoter. As we will see, these can actually represent many other aspects of regulatory complexes. We consider an arbitrary number $N$ of TFs, noted $f \in \mathscr{F}$

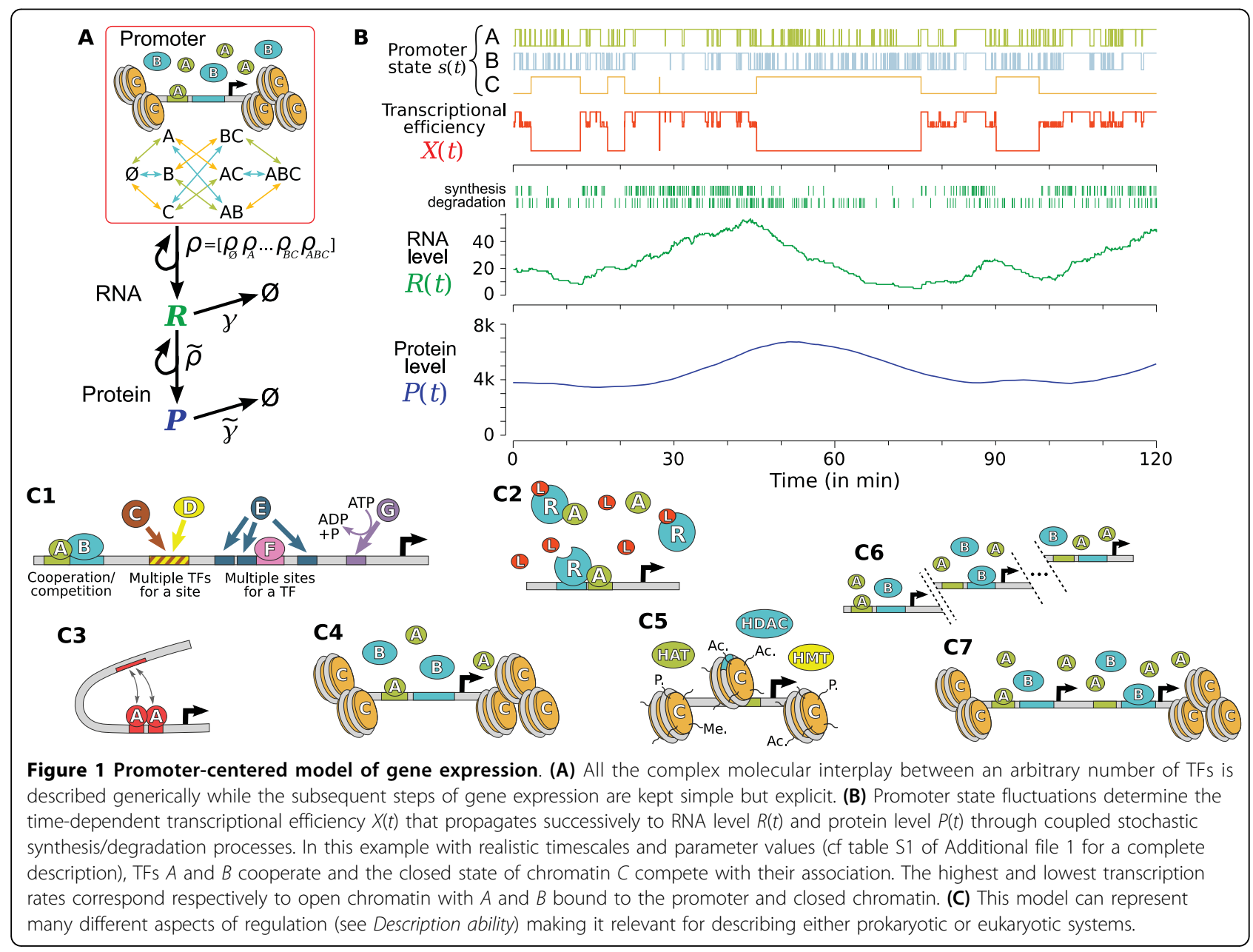


(eg. $\mathscr{F}=\{A, B, C, \ldots\})$. The set of TFs that are bound to the promoter at a given instant ( $2^{N}$ possible combinations) is referred to as the promoter state and noted $s \in\{\varnothing, A, B$, $A B, C, A C, \ldots\}$. Classically, a TF $A$ at concentration $[A]$ binds and unbinds on/from its target site with rates $[A]$ $k_{\text {on }}$ and $k_{\text {off }}$ respectively. However, because of cooperation and competition [56-61], the association and dissociation constants $k_{\text {on }}$ and $k_{\text {off }}$ of any TF actually depend on the combination of all the other TFs present on the promoter. We define the $N \times 2^{N}$ matrix $\mathbf{k}^{0}$ summarizing the association and dissociation constants of each of the $N$ TFs and for each of the $2^{N}$ states. $k_{f, s}^{0}$ describes the transition from state $s$ to state $s \ominus f$ (where $\ominus$ denotes the symmetric difference between sets; eg. $A B C \ominus B=A C$ and $A C \ominus B=$ $A B C)$. Multiplying each association rate by the concentration $[f]$ of the TF that binds, we obtain the $N \times 2^{N}$ matrix k describing all the transition rates of the weighted directed graph of promoter states (figure 1A). To focus on gene-intrinsic stochasticity, any source of gene-extrinsic stochasticity is avoided by considering TFs to be uniformly distributed in space and in constant concentration, so that the $N$-vector $[f]_{f \in \mathscr{F}}$ of TFs' concentrations is a parameter of the model. This generic description can represent arbitrarily complex relations of combinatorial cooperation/ competition and kinetic influence.

Although for simplicity this short description of the model as well as the examples in this paper do not consider TFs associating and dissociating simultaneously as a complex (eg. $\varnothing \rightleftarrows A B$ ), the model can actually account for these transitions (cf Additional file 1, $\mathbb{\$}$ ). The influence of considering such reactions is discussed (cf Discussion).

Combinatorial cooperation/competition also takes place in RNA polymerase recruitment and provide each promoter state with a certain competency to initiate transcription $[48-50,56,61,65,66]$. This is described more accurately than the binary view of on/off promoter activity, by a $2^{N}$-vector $\rho$ of state dependent transcriptional efficiency.

\section{Subsequent steps of gene expression}

As in most models of stochastic gene expression, RNA and protein levels (noted $R(t)$ and $P(t)$ respectively) follow classical stochastic birth-and-death processes with instantaneous and first order reactions (cf figure 1A). The time-dependent transcriptional efficiency of promoter $X(t)$ determines the synthesis rate of RNA molecules which, in turn, degrades with rate $\gamma$. Protein level $P(t)$ is driven by the translation of RNA molecules at rate $\tilde{\rho}$ and a degradation rate $\tilde{\gamma}$ (figure 1B). Implications of the very usual simplifications of instantaneous transcription and translation and first order degradations will be discussed (see Discussion).

\section{Energetic signification}

Regulation is classically approached with thermodynamic methods $[79,80]$. We show that our model can be expressed in energetic terms and constitute a generalization of these approaches by extending the range of systems that can be represented (ie. including energyconsuming systems such as eukaryotic promoters) and the type of metrics that can predicted (ie. including measures of dynamic and stochastic properties). The usual thermodynamic formulation of cooperative and competitive association/dissociation of TFs $[67,68,79,80]$ is equivalent to assign a Gibbs free energy to each promoter state. For our system, it corresponds to a $2^{N}$-vector $\mathbf{G}^{\mathbf{0}}$ in the standard condition (ie. all TFs having unit concentration. For arbitrary concentrations, $G_{s}=G_{s}^{0}+$ $\mathrm{k}_{\mathrm{B}} T \Sigma_{f \notin s} \log [f] . T$, temperature; $\mathrm{k}_{\mathrm{B}}$, Boltzmann constant). This representation allows one to predict the equilibrium steady-states (by applying a Boltzmann factor) and has been widely used to investigate the mean aspects of prokaryotic regulation $[79,80]$. But it has the drawback to restrict the analysis to energetically-closed systems and, not carrying any kinetic information, it forbid any investigation of the stochastic aspects of expression.

For this energetic formulation to be equivalent to the kinetic one, we have to consider an additional set of energy values that are difficult to access experimentally [68], namely the energy of the activation barrier for each reaction. Representing them by the $N \times 2^{N}$ matrix $\mathbf{E}^{\mathbf{0}}$, the energy that must be overcome for reaction $s \rightarrow s \ominus f$ to occur is $E_{f, s}^{0}-G_{s}^{0}$. The kinetic constants $\mathbf{k}^{0}$ can then be obtained as $k_{f, s}^{0}=e^{-\left(E_{f, s}^{0}-G_{s}^{0}\right) / k_{\mathrm{B}} T}$. This reformulation allows us to explicitly make the distinction between open and closed systems (ie. involving or not energy-dependent reactions). For a closed system, nothing else than TFs and promoter DNA are involved and the energy of the activation barrier is the same in both directions of each reaction $s \rightleftarrows s \ominus f$ so that $E_{f, s}^{0}=E_{f, s \ominus f}^{0}$. For an open system, energy-dependent reactions (eg. involving ATP hydrolysis) are possible, resulting in $E_{f, s}^{0} \neq E_{f, s \ominus f}^{0}$ (the difference being the energy received by the system). It can be shown that it is only in the case of an open system that the transition graph (figure 1A) can contain directed cycles so that the detailed balance property of the chemical system (corresponding to the reversibility property of the underlying Markov chain) does not hold [75] (cf Additional file 1, $\$ 2.2$ ). This property has meaningful biological implications in the context of promoter dynamics and is most likely an essential feature of eukaryotic promoters (see Results).

\section{Description ability}

Many biologically relevant features of regulatory systems can be easily represented with this generic model only as a matter of parametrization. In particular, it can account for multiple TFs competing for the same binding site or a TF having multiple binding sites (figure $1 \mathrm{C} 1$ ). The 
general formulation of the model (cf Additional file 1, $\$ 2$ ) allows one to represent the association/dissociation of molecules either on their own or within complexes of various composition. This is an essential aspects of most ligand-receptor regulated genes (figure $1 \mathrm{C} 2$ ) where the ligand modifies the receptor's affinity with DNA and ability to recruit different cofactors. Moreover, what was so far considered a TF molecule bound or not can be generalized to represent other aspects of the state of a promoter: Alternative conformational states (DNA looping, chromatin open/closed state, nucleosome sliding ...) and the status of histone tail residues (figures 1C3-5). Furthermore, these epigenetic factors can be represented to be modified by explicit remodeling complexes and histone modifying enzymes, taking into account their essential energy-dependent and nearly irreversible nature. Also, the affinity and the enzymatic/remodeling activity of any molecule can be defined to depend on these various epigenetic factors in a combinatorial way in order to account for all the potentiality of the histone code. For instance, one can set parameters so that the acetylation of a given histone tail residue can only occur in the presence of a given histone acetyletransferase (HAT) with specific cofactors and corresponds to an highly energetic reaction. The property of epigenetic changes and promoter occupancy by TFs to occur cyclically on eukaryotic promoters $[49-52,66]$ can be represented with various sorts of deviations from an ideal sequential recruitment (ref [71] and case (ii) in Overview of derivations and Additional file 1, \$3.1). In the Results section, we show that this behavior is directly due to the energy-dependent modifications of chromatin that our model can represent. Other situations of interest can be described such as multiple copies of the same gene (figure 1C6) or two genes in the same chromatin context (figure 1C7), reproducing a situation that has demonstrated experimentally that chromosome positioning and chromatin dynamics is a key factor of noise in eukaryotic gene expression $[5,6,10]$.

\section{Overview of derivations}

Here we provide a short description of the theoretical derivations of this paper. For details, the reader can refer to the Additional file 1 (\$3).

Several generic approaches have been proposed for deriving steady-state and/or spectral indicators of the stochastic activity of an arbitrary (sometime only linear) reaction network $[23,34,69,70,74,75]$. These techniques are based on a reformulation of the chemical master equation (CME) in terms of moments or on its approximation into a Langevin equation which is then solved by different methods (eg. linear noise approximation, frequency domain analysis, ...). These powerful methods could be applied to our system and may lead to similar expression of noise power as ours. However, the methods we employ here makes no approximation of the CME, thus providing exact results. It takes advantage of the fact that the CME for the whole system can be decomposed into a (finite) promoter-CME from which RNA and protein levels fluctuations are deduced using inhomogeneous Poisson birth-and-death processes. The promoter-CME is solved as a continuous-time Markov chain with standard eigenvalues-decomposition techniques [75] that have the great advantage to result in expressions of power spectra and normalized variances in terms of simple elementary components that reveal the structure of the dynamics. Note that the application of the previously mentioned generic methods on an arbitrary system as the one we consider would also require to solve a linear system or an eigenproblem.

Previous models of gene expression that incorporate a detailed description of promoter states were either solved for the steady-state mean [73] or moments [42] of expression or simulated with a Gillespie algorithm $[52,67,68,76,78]$. Although these simulation studies provided valuable insights into the dynamic aspects of promoter stochastic activity, the lack of analytical results do not allow for complete understanding of the dynamics and extensive exploration of parameters. Thus the dynamic aspects of promoter stochastic activity (eg. as measured by power spectra or autocorrelations) remain partially unexplored from the modeling point of view and the steady-state ones were not described in terms of distribution (a metric that reveals essential features such as number/position/size/shape of modes). Here, we derive both power spectra and distributions for an arbitrary regulatory structure.

In our model, the time-dependent vector $\varphi(t)$ describing the probability for a promoter to be in each state evolves according to the CME

$$
\frac{\mathrm{d} \varphi(t)}{\mathrm{d} t}=\mathbf{M} \varphi(t)
$$

where the $2^{N} \times 2^{N}$-matrix $\mathbf{M}$ represents the state-tostate transition rates (constructed from $\mathbf{k}$ in the simple version of the model and provided directly as a parameter in the general version). Diagonal elements of $\mathbf{M}$ are set so that $M_{s, s}=-\sum_{s^{\prime} \neq s} M_{s^{\prime}, s}$.

The forward solution $\varphi(t+\tau)$ of the CME is simply obtained from the decomposition of $\mathbf{M}$ into eigenvalues $\lambda_{i}$ and eigenvectors $\Lambda_{i}$. Combined with $\rho$, it gives the autocorrelation of process $X(t)$

$$
\langle X(t) X(t+\tau)\rangle_{t}=\sum_{i} e^{\lambda_{i}|\tau|}\left[\sum_{s} \rho_{s} \Lambda_{s, i} \sum_{s^{\prime}} \Lambda_{i, s^{\prime}}^{-1} \rho_{s^{\prime}} \Lambda_{s^{\prime}, 0}\right]
$$

from which is deduced the power spectrum $S_{X}(\omega)$ (Eq 5). 
In the general case, the eigendecomposition of $\mathbf{M}$ is obtained numerically, but we provide analytical expressions in the case of (i) a two-states promoter and (ii) a homogeneous isolated directed cycle with backward reactions, ie. a cycle of $n$ states noted

$$
s_{0} \underset{k^{\mathrm{b}}}{\stackrel{k^{\mathrm{f}}}{\rightleftarrows}} s_{1} \underset{k^{\mathrm{b}}}{\stackrel{k^{\mathrm{f}}}{\rightleftarrows}} s_{2} \ldots s_{p} \ldots s_{n-1} \underset{k^{\mathrm{b}}}{\stackrel{k^{\mathrm{f}}}{\rightleftarrows}} s_{0}
$$

In that case, the eigendecomposition

$$
\left\{\begin{array}{l}
\lambda_{i}=k^{\mathrm{f}}\left(e^{\frac{-2 \pi j}{n} i}-1\right)+k^{\mathrm{b}}\left(e^{\frac{2 \pi j}{n} i}-1\right) \\
\Lambda_{s_{p}, i}=e^{p \frac{2 \pi j}{n} i}
\end{array}\right.
$$

shows that the spectrum of $\mathbf{M}$ consists of $n$ eigenvalues regularly spaced on a circle tangent to the imaginary axis at 0 for an irreversible cycle $\left(k^{b}=0\right)$. It flattens as an ellipse toward the real axis as backward reactions $k^{b}$ increase. Therefore the coherence of the periodic dynamics increases with the number of steps and the ratio $k^{f} / k^{b}$.

The steady-state rate of free energy change $\dot{E}$ (Eq 8) is obtained by noticing that any reaction $s \rightarrow s$, occurs at a rate $\Lambda_{s}, 0 M_{s}{ }^{\prime}, s$ and results in a change of free energy of $\mathrm{k}_{\mathrm{B}} T \log M_{s^{\prime}, s} / M_{s, s^{\prime}}$.

Power spectra $S_{R}(\omega)$ and $S_{P}(\omega)$ are obtained by remarking that $R(t)$ (resp. $P(t)$ ) is a birth-and-death process with birth rate being an inhomogeneous Poisson process with instantaneous rate $X(t)$ (resp. $\tilde{\rho} R(t)$ ). Normalized variances $\sigma_{\mathrm{X}}{ }^{2} /\langle\mathrm{X}\rangle^{2}, \sigma_{\mathrm{R}}{ }^{2} /\langle R\rangle^{2}$ and $\sigma_{P}{ }^{2} /\langle P\rangle^{2}$ are deduced from power spectra using the property $\left\langle\xi^{2}\right\rangle=\int_{-\infty}^{+\infty} S_{\xi}(\omega) \mathrm{d} \omega / 2 \pi$.

Distributions of RNA levels are obtained by solving numerically the system of equations corresponding to the steady-state of the CME that considers both the promoter and the RNA level

$$
\mathbf{D}_{\boldsymbol{\rho}}\left(\varphi_{r-1}-\varphi_{r}\right)+\gamma\left((r+1) \varphi_{r+1}-r \varphi_{r}\right)+\mathbf{M} \varphi_{r}=0
$$

where $\mathbf{D}_{\boldsymbol{\rho}}$ is the diagonal representation of $\boldsymbol{\rho}$ and the $s^{\text {th }}$ element of $\varphi_{r}$ is the probability to have $r$ RNA molecules and the promoter in state $s$.

\section{Results}

With this model that can represent arbitrarily complex regulatory system from prokaryotes to eukaryotes, we show that the molecular interplay can cause a single gene with constant concentrations of TFs to demonstrate various forms of complex activity (eg. multiple periodicities, relaxation times) that are usually found at the network-level. We describe these activities in relation with experimental observations from the literature and highlight underlying mechanisms that might cause them and properties that they might provide.

\section{Spontaneous activity of an arbitrary regulatory structure}

We show that the power spectrum $S_{X}(\omega)$ of transcriptional efficiency $X(t)$ (figure 2A1) can be simply written as the sum of $2^{N}$ simple elementary components (ie. elementary signals or modes)

$$
S_{X}(\omega)=\sum_{i} \frac{-2 \lambda_{i} \beta_{i}^{2}}{\lambda_{i}^{2}+\omega^{2}}
$$

With $\beta_{i}=\left[\sum_{s} \rho_{s} \Lambda_{s, i} \sum_{s^{\prime}} \Lambda_{i, s^{\prime}}^{-1} \rho_{s^{\prime}} \Lambda_{s^{\prime}, 0}\right]^{1 / 2}$ from the decomposition of the transition rate matrix $\mathbf{M}$ into eigenvalues $\lambda_{i}$ (a set of points on a plane; figure 2B) and eigenvectors $\Lambda_{i}$.

Each eigenvalue $\lambda_{i}$ that lays on the real axis (ie. abscissa axis of the plane on figure $2 \mathrm{~B}$ ) corresponds to an aperiodically fluctuating signal with characteristic relaxation time $-1 / \lambda_{i}$ determined by its position on the axis (the further from 0 , the faster the fluctuations). For instance, the purple eigenvalue on figure $2 \mathrm{~B}$ corresponds to the purple component on figure 2A1. Eigenvalues that are not on the abscissa axis come in pairs of conjugates (ie. symmetric with respect to this axis) and correspond to periodic stochastic signals which oscillating frequency $\left|\operatorname{Im}\left(\lambda_{i}\right) / 2 \pi\right|$ is given by the ordinate of the eigenvalue (eg. light blue point on figure $2 \mathrm{~B}$ and light blue curve on figure 2A1). The thinness of the peak in the power spectrum of the component is an important characteristic of the oscillatory behavior. It is described by the coherence factor

$$
\left|\frac{\operatorname{Im}\left(\lambda_{i}\right)}{2 \pi \operatorname{Re}\left(\lambda_{i}\right)}\right|
$$

that corresponds intuitively to the number of oscillations after which two initially synchronized promoters are significantly desynchronized. This can be directly measured on experimental data such as obtained by chromatin immunoprecipitation (ChIP) $[49,50,52,66]$, laser crosslinking [51] and fluoresence microscopy techniques [48]: the damping of the oscillations upon synchronous activation of a large number of identical promoters reflects the desynchronization among the population due to the stochastic timing in each individual cell [52]. Gray lines on figure $2 \mathrm{~B}$ represent a coherence factors of 0.5 and 1 . The most coherent eigenvalues (light blue) have a coherence of $\sim 1$.

It is important to mention that the repartition of eigenvalues on the complex plane (figure 2B) - called 

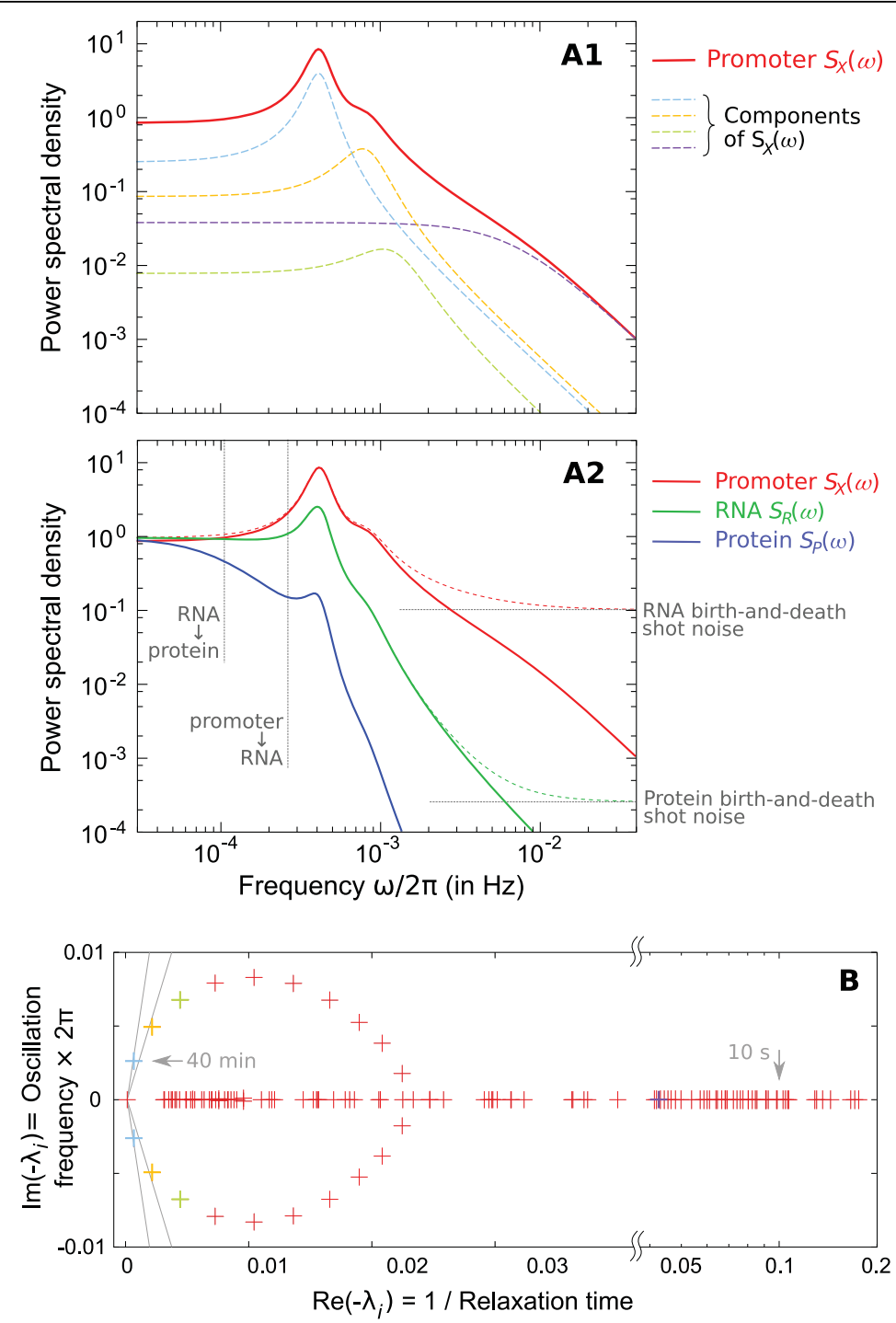

Figure 2 Portrait of the regulatory structure dynamics and its transmission to RNA and protein levels. (A1) The power spectrum $S_{X}(\omega)$ of the transcriptional efficiency process $X(t)$ (red curve) is the sum of simple components (dashed curves). (A2) These fluctuations of transcriptional efficiency are transmitted to RNA and protein levels undergoing at each step (cf Eq. 6) the addition of a shot noise due to finite synthesis/ degradation events (horizontal gray dashed lines: noise levels) and a low pass filtering due to time averaging (vertical gray dashed lines: cutoff frequencies). Dashed red and green curves are intermediate spectra $2 \chi(R\rangle+S_{X}(\omega)$ and $\tilde{\rho}^{2}$ illustrating the effect of the shot noise. (B) Each eigenvalue $\lambda_{i}$ of matrix $\mathbf{- M}$ (or pair of conjugates) corresponds to an elementary component (or mode) in (A1) and determines its characteristics (eg. frequency and thinness of the peak). For instance, the arrows correspond to a 40 min oscillation period and a $10 \mathrm{~s}$ relaxation time. Colored crosses identify the components displayed in (A1). Many observables on the promoter can be described by the spectrum of $-\mathbf{M}$ (cf text), making it an accurate representation of the whole regulatory structure dynamics.

the spectrum of matrix $\mathbf{M}$ - provides a picture of the whole dynamics of the promoter. Indeed, the power spectrum of any observable (including the presence/ absence of a given TF, the co-occurrence of two TFs, the contact between two chromatin proteins ..., as observed by techniques such as single-molecule FRET $[81,82]$ for instance) can be derived similarly to $S_{X}(\omega)$ (ie. using different values for $\rho_{s}$ ) and results from the same set of eigenvalues.
Interestingly, the spontaneous activity of a single-gene with all input constant (Eq 5 and figure 2A1) is very similar to what has been indentified at the network-level $[30,34]$ considering the interaction of several (simpler) genes. This shows that single nodes of gene regulatory networks can be much more complex than they are usually considered.

The example provided in figure 2 to illustrate the generic decomposition Eq 5 aims at reproducing the 
behavior of an eukaryotic promoter with realistic parameters and timescale relationships. Experimental studies of eukaryotic promoter dynamics reveal slow oscillatory patterns of occupancy and chromatin modifications with a period in the order of few tens of minutes $[48-50,52,66]$ and a rapid turnover of TFs in the order of tens of seconds [45-48]. The transition rates $\mathbf{k}^{\mathbf{0}}$ were obtained using a stochastic optimization algorithm that promotes a coherent oscillatory behavior at a timescale significantly slower than the rest of the dynamics, based on criteria regarding the spectrum of $\mathbf{M}$ and with concentration of TFs set in the physiological range for eukaryotes [52,83,84], ie. $3 \mathrm{nM}$ (cf table S4 in Additional file 1 for details). The resulting system demonstrates a strong cyclic activity with a period of $40 \mathrm{~min}$ while the fastest events occur at a timescale of less than $10 \mathrm{~s} \mathrm{(cf}$ arrows on figure 2B). However, these fast events do not necessarily correspond to TF residence times and the question of how a slow global activity arises from the rapid dynamics of the molecules remains open. Most propositions from the literature suggest that at least some of these factors (eg. chromatin modifications) are slow [48,52-54].

Within the framework of our model, the particular ideal system consisting in a cycle of homogeneous directed transitions (as in [71]) produces a circle of eigenvalues in the spectrum of $\mathbf{M}$ ( $\mathrm{cf}$ Additional file 1, \$3.1.1), similarly to what results from the optimization algorithm (figure 2B). The shape and number of points of this circle indicate that the promoter progresses along a 22 -steps cycle of strongly directed transitions. In comparision, [52] supposes a priori a cycle of 6 of such transitions and is also able to reproduce oscillations with simulations. Note that the further development regarding energy consumption we will make in this paper also argue for the physical realism of the system of figure 2 .

We described the spontaneous fluctuations of promoter activity for any arbitrary promoter by a modal decomposition of the power spectrum $S_{X}(\omega)$ (Eq 5) and illustrated it with an eukaryotic example (figure 2). Now we will focus on how these fluctuations propagate through the RNA and protein levels.

\section{Transmission of promoter stochasticity to RNA and protein levels}

Both RNA and protein levels $R(t)$ and $P(t)$ follow an inhomogeneous birth-and-death process which inhomogeneous birth rates are $X(t)$ and $\tilde{\rho} R(t)$ respectively. From this scheme, we derive the power spectra of RNA and protein levels (resp. $S_{R}(\omega)$ and $S_{P}(\omega)$ ) from $S_{X}(\omega)$ (cf Additional file 1, \$3.3):

$$
\begin{aligned}
& S_{P}(\omega)= \\
& {[\overbrace{2 \tilde{\gamma}\langle P\rangle}^{\text {Protein }}+\tilde{\rho}^{2}[\underbrace{\begin{array}{c}
\text { RNA } \\
\text { shot noise }
\end{array} \underbrace{2 \gamma\langle R\rangle}+\overbrace{S_{X}(\omega)}^{\text {Promoter }}]}_{=S_{R}(\omega)} \overbrace{\left(\gamma^{2}+\omega^{2}\right)^{-1}}^{\text {Promomamics }}] \overbrace{\left(\tilde{\gamma}^{2}+\omega^{2}\right)^{-1}}^{\text {low-pass filter }}}
\end{aligned}
$$

with $\langle P\rangle=\langle R\rangle \tilde{\rho} / \tilde{\gamma},\langle R\rangle=\langle X\rangle / \gamma$ and $\langle X\rangle=\beta_{0}$. This expression clearly separates the different gene-intrinsic stochasticities and their physical principles from a dynamic viewpoint. In agreement with previous analyzes $[34,69]$, each of the two steps (transcription and translation) result in (i) the addition of a shot noise (the classical low-copy Poisson noise) due to the finite number of random birth-and-death and determined by RNA and protein abundance and (ii) a low-pass filtering due to time averaging determined by RNA and protein lifetimes (figure 1A2).

Transcript and protein life-times, as reported by global analyzes on the yeast transcriptome [85] and proteome [86] for instance, are broadly distributed: from $3 \mathrm{~min}$ to $90 \mathrm{~min}$ for RNA half-lives and from less than $4 \mathrm{~min}$ to more than a day for protein half-lives. Interestingly, in regard with the typical period of 40 60 min of promoter cycling observed in vivo [48-50,52], these life-times can either dampen the promoter oscillations or let it propagate up to the protein level. Indeed, several studies showed that the RNA level can display the same cyclical patterns as observed on the promoter [48,52]. In the example of figure 1 , transcript and protein life-times were chosen rather short (namely $1 / \gamma=10 \mathrm{~min}$ and $1 /$ $\tilde{\gamma}=25 \mathrm{~min}$ ) and oscillation can be observed clearly in RNA fluctuations and slightly in protein fluctuations. Another global analysis in yeast [84] showed that RNA abundance ranges from less than 1 copy per cell to a hundred and that proteins are typically a thousand times more abundant. In figure 1A2, where RNA and protein abundances are $\langle R\rangle \simeq 30$ and $\langle P\rangle=1000\langle R\rangle$, the oscillations are more important than the shot noises are. Approaching 0 for RNA abundance increases the shot noise level with respect to promoter fluctuations $S_{X}(\omega)$ and leads to the regime where the noise of RNA birthand-death dominates. 
Noticeably, the derivation from power spectrum $S_{P}(\omega)$ of the normalized variance (a more synthetic but less descriptive indicator) reads (cf Additional file 1, $\$ 3.3$ ):

$$
\begin{aligned}
& \frac{\sigma_{P}^{2}}{\langle P\rangle^{2}}=
\end{aligned}
$$

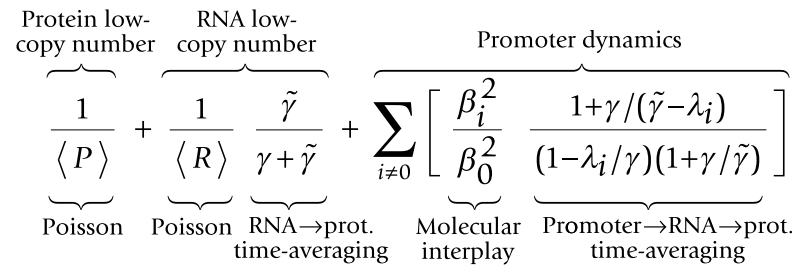

an expression similar to the well-known expression due to J. Paulsson [23] who first provided such a clear separation, but considered a set of independent twostates genes. The time-averaging coefficients and Poisson $1 /\langle P\rangle$ terms directly result from the filters and shot noise terms in $S_{P}(\omega)$.

While power spectra describe the dynamic aspects of fluctuations (eg. periodicities, relaxation times), it cannot capture several important features that reside in steadystate distributions. We provide here a numerical method for finding the full steady-state distribution of RNA molecules without any further simplification to the model (cf Additional file 1, $\$ 3.3$ for details). A previous analysis proposed a method for finding the set of moments with a similar generic model for the promoter [42], but this does not reveal meaningful features such as multimodality and position/size/shape of the different modes as it will be illustrated in figures $3 \mathrm{C}$ and $4 \mathrm{C}$.

Power spectra, normalized variance and full steadystate distribution are complementary indicators that, being derived prior to instantiation of the model, provide a comprehensive description of any arbitrarily complex regulatory system.

\section{Cyclical promoter occupancy and remodeling, periodic activity and energy-dependence}

Our system is able to reproduce the strongly periodic activity that is observed experimentally on eukaryotic promoters $[48-52,66]$ and referred to as cyclical recruitment or loading profile (figure 2A). This arises when the graph of promoter states contains directed cycles (ie. a closed path over which the product of kinetic constants is not the same in both directions). The very few previous works also accounting for this oscillating behavior with different modeling frameworks $[52,71,72]$ indeed relied on a similar mechanism. We show that the absence of directed cycle in closed systems imposes that all eigenvalues are on the real axis, thus forbidding any oscillation (cf Additional file 1, $\$ 2.2$ and 3.2). The signature of cycles are circles of eigenvalues in the spectrum of matrix $\mathbf{M}$ (cf Overview of derivations and Additional file $1, \$ 3.1$ ), as in figure $2 \mathrm{~B}$ for instance. As also remarked by $[52,71]$ but in less quantitative and/or generic terms, the essential resulting property is that the longer, the more directed and the more homogeneous the cycle, the more coherent the periodic activity.

The energetic formulation of the model (cf Methods and Additional file $1, \$ 2.2$ ) show that directed cycles are necessarily due to energy-dependent transitions in promoter state and that the more energy is consumed along the cycle, the more directed it is. The well-known property that energy-dependence is required for oscillations in metabolic networks [87] (that considers varying amount of several species in large quantities interacting through deterministic laws) can be transposed to our case (that considers the stochastic activity of a single promoter with all TFs concentration constant).

This observation has important consequences on our understanding of promoter dynamics and stochasticity. Indeed, the cyclical occupancy and remodeling profiles observed on eukaryotic promoters is highly coherent [48,50-52,66] (ie. when a large number of promoters are synchronized, it takes many oscillations before they are significantly desynchronized). In the light of our results, such a coherence requires the presence of cycles that (i) span over a very large number of states (likely several hundreds for the most coherent cases) and (ii) are highly energy-consuming. This first point is strongly supported by the profusion of possible promoter states provided by the combinatorial patterns of histone tails modifications and nucleosome positioning [60-64] and the alternate compositions of multiprotein complexes found on promoters $[53,54]$. The second point comes in good agreement with the fact that many ATP-dependent chromatin remodeling complexes (eg. SWI/SNF and NuRD complexes) and histone modifying enzymes (that use a cofactor as an energy donor) actively participate in the cycling behavior of active promoters [50,51]. Moreover, ATP depletion has been observed to totally suppress oscillations [51]. Hence, the oscillatory activity observed on eukaryotic promoter cannot be explained without energy consumption, a feature indeed widely present on these promoters.

The very stereotyped case consisting in a cyclic sequence of homogeneous irreversible transitions $\left(k^{\mathrm{b}}=\right.$ 0 in case (ii) of Overview of derivations and Additional file $1, \$ 3.1$ ) results in a highly coherent periodic dynamics. But, considering reactions irreversible, this 

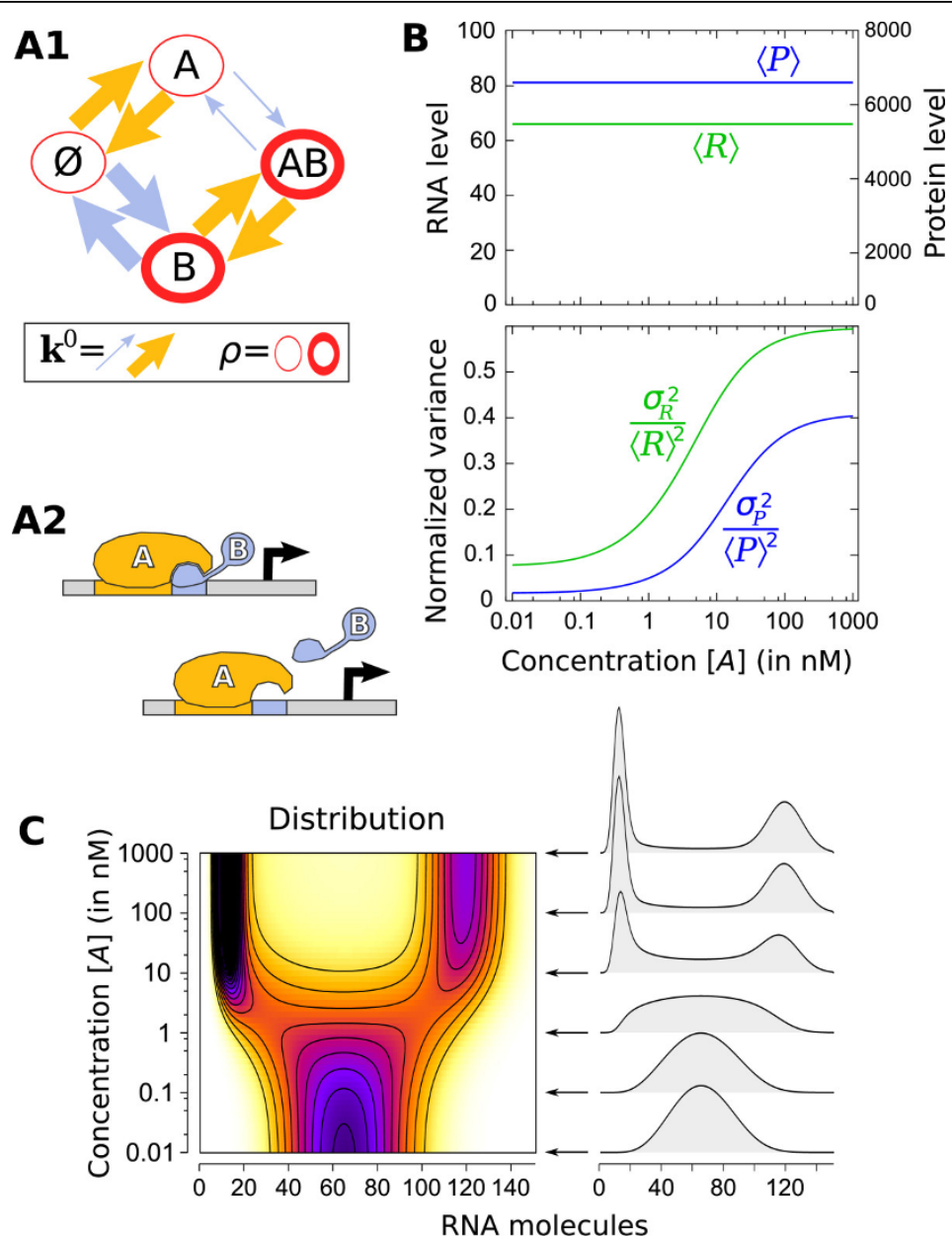

Figure 3 Stochasticity induced at high concentration of a TF. This minimalist system (A1) (see text or table 2 of Additional file 1 for description) that can correspond to simple molecular scenarios (A2) demonstrates that, contrarily to a common idea, increasing the concentration of a TF can result in a larger variability (B). A more precise picture of this phenomenon is provided by observing how distribution changes with TF concentration (C). Robustness of this behavior with respect to deviations from this ideal minimal model are presented in figures $\mathrm{S} 1$ and S2 of Additional file 1.

system theoretically consumes an infinite amount of energy. Also, in this ideal system, events of recruitment are perfectly ordered and sequential (ie. sequence is deterministic). Away from this unrealistic case, when the whole set of kinetic constants of the promoterstate graph contains directed cycles with heterogeneous transitions, alternative pathways (of various length and duration) and includes backward, incoming and outgoing transitions then the system can still demonstrate a periodic activity (ie. non-real eigenvalues). Similarly to the "preferentially random" scheme of [52], although events do not follow a predefined sequence and display a significant level of randomness, they can still tend to occur in a preferred order and result in a periodic global pattern. As a result, organizing the molecular interplay to provide the promoter with a structured dynamics (the ideas of "transcription clock" [50] and "molecular memory" [41]) has a clear energetic cost. The relevant measure reflecting this fundamental cost is the steady-state energy consumption rate:

$$
\dot{E}=\mathrm{k}_{\mathrm{B}} T \sum_{s} \Lambda_{s, 0} \sum_{f} k_{f, s} \log \left[k_{f, s} / k_{f, s \ominus f}\right]
$$

Indeed, during the optimization process used to obtain the example of eukaryotic promoter, the energy consumption rate $\dot{E}$ increases along with the coherence. For the system shown in figure $2, \dot{E}$ equals $0.05 \mathrm{k}_{\mathrm{B}} T . \mathrm{s}^{-1}$. The period of oscillations being $40 \mathrm{~min}$ and considering $20 \mathrm{k}_{\mathrm{B}} T$ for an ATP hydrolysis in physiological conditions [88], this energy consumption corresponds to the equivalent of $\sim 6$ ATP hydrolyzes per cycle. This is reasonable although real promoters are actually larger, more coherent and their energy consumption is certainly more important [50]. 


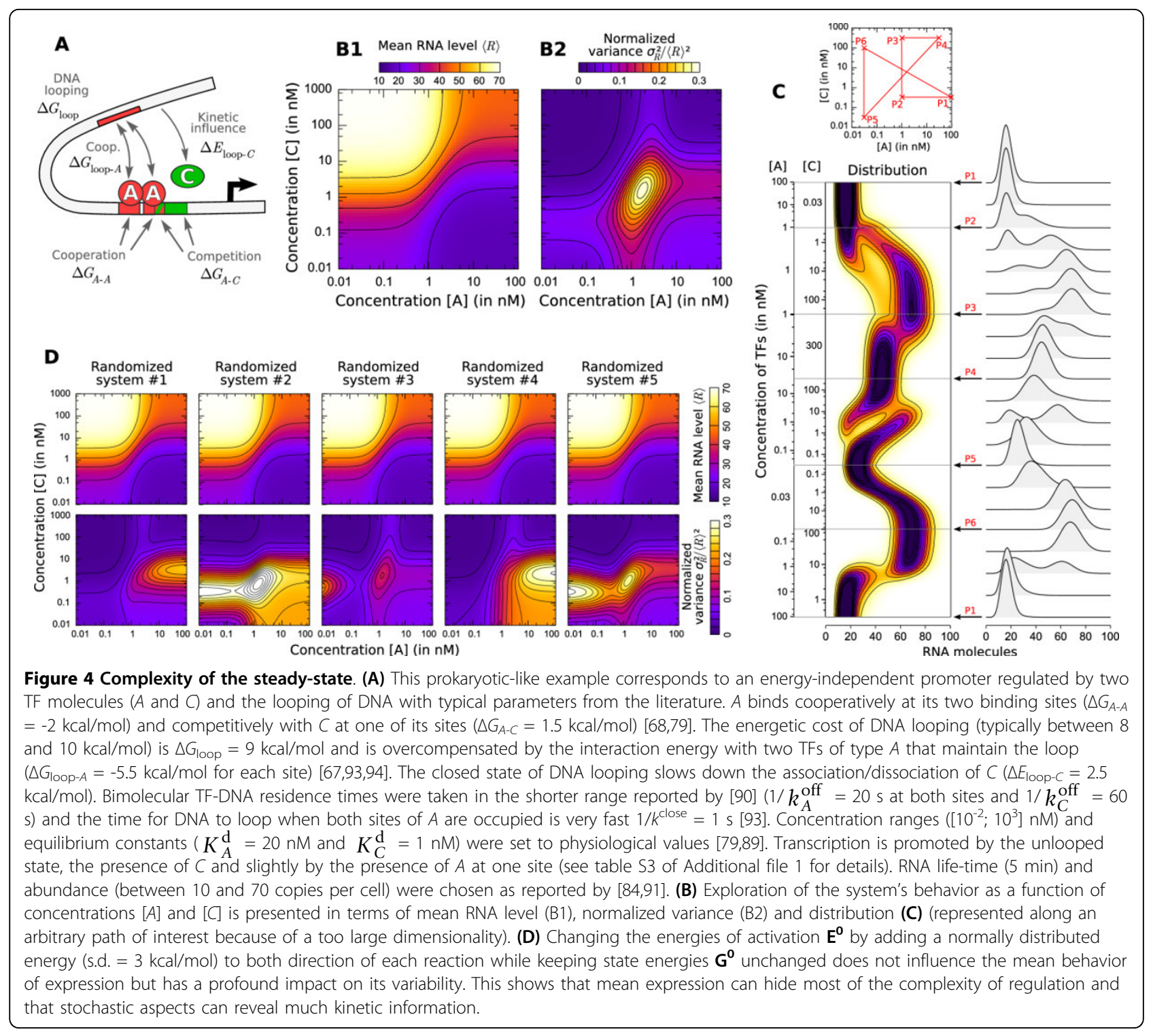

However, although necessary, energy-dependence is not sufficient for a periodic activity and is not the sole factor influencing the extent of this phenomenon (cf Additional file 1, $\$ 3.2$ ). Hence, the correlation between structured promoter activity and energydependence is not direct, raising questions about their probably non-trivial evolutionary relation (see Discussion).

\section{Stochasticity can be induced by a high TF concentration} One possible use of this model is to identify a behavior of interest, isolate its minimal requirements and then test its applicability with realistic parameters and on larger and more biologically plausible systems. We give here an example of such use of the model.
As stochasticity is often due to the rareness of some discrete events, it is very commonly associated to low concentrations. However, we show that, in some situations, stochasticity can on the contrary be induced by a high concentration of a TF. The simplest system for this behavior to occur is described on figure $3 \mathrm{~A} 1$. It consists of a promoter with two TFs $(A$ and $B)$ where the transcription rate only depends on $B$. The only mutual influence between TFs is that $B$ associates and dissociates more slowly when $A$ is bound to the promoter. We used realistic parameter values similar to those reported by quantitative thermodynamics $[79,89]$ and single-molecule kinetics studies [90] of bacterial regulation for concentrations $\left([A] \in\left[10^{-2} ; 10^{3}\right] \mathrm{nM}\right.$ and $[B]=5 \mathrm{nM})$, for bimolecular TF-DNA residence times 
$\left(1 / k_{A}^{\text {off }}=30 \mathrm{~s}\right.$ and $\left.1 / k_{B}^{\text {off }}=60 \mathrm{~s}\right)$ and equilibrium constants $\left(K_{A}^{\mathrm{d}}=0.5 \mathrm{nM}\right.$ and $\left.K_{B}^{\mathrm{d}}=5 \mathrm{nM}\right)$ and for modification of activation energy upon interaction $(\Delta E=2.5$ $\left.\mathrm{kcal} . \mathrm{mol}^{-1}\right)$. RNA and protein life-times are 5 and 20 min [91]. All parameters are summarized in table S2 of Additional file 1.

This simple system demonstrates not only that a TF can regulate the variance of RNA and protein levels without influencing their mean (as previously identified with various mechanisms $[28,42]$ ), but more interestingly, that normalized variance can increase with the concentration of a TF (figure 3B). Indeed, here it is at high concentrations of $A$ that events influencing transcription (ie. $B$ associations and dissociations) become rare and, as it has been known for a long time [2,3,14,23-27,30,32,34-37,40], slow promoter dynamics result in strong variability. RNA distribution goes from unimodal to bimodal as $[A]$ increases (figure $3 \mathrm{C}$ ). Thus, the oversimplistic assumption that increasing the concentration of a TF necessarily reduces the stochasticity is not always valid.

Moreover, this property appears to be quite robust when exploring all sorts of deviations from this ideal case (eg. considering a different concentration for $[B]$, an influence of $B$ on $A$, a dependency of the transcription rate on $A \ldots$, figure $\mathrm{S} 1$ in Additional file 1), and even for a more complex regulatory system with a larger number of TFs and randomly drawn parameters (figure S2 in Additional file 1).

Simple molecular scenarios can be imagined that would give rise to this behavior. For instance, the shape of a TF (type $A$ ) can be so that, when bound, it prevents other TFs (type $B$ ) from association/dissociating from the promoter (figure 3A2). Another example would be to consider $B$ the chromatin state and $A$ a TF that binds to the same site as chromatin remodeling complexes. Then, designing molecular constructions to verify this hypothesis experimentally appears to be promising.

\section{Potentiality of the molecular interplay}

Using this generic model and the prediction of the different indicators we provided allows us to explore in detail the activity of regulatory systems on large ranges of concentration. To illustrate the potentiality of steadystate and dynamic properties, we consider here two examples (figures 4 and 5) dedicated to represent respectively a prokaryotic promoter - with typical features as in $[56,67,80]$ - and a eukaryotic promoter (the same as in figure 2) - reproducing the periodic behavior as observed in $[48-52,66]$. Although it is the expected behavior that complex activities can arise from large systems with many parameters, we show that such behavior can occur with physically realistic parameters and be due to relevant biological mechanisms. We further argue in Discussion that several typical features of eukaryotic promoter are precisely those that give rise to a complex dynamics. We also show how this complexity is hidden by common measures and/or modeling frameworks and identify what kind of features can modulate or constrain it.

\section{Steady-state aspects}

Representing the behavior of a gene as a function of TF concentrations by a combination of Hill functions is very common and often matches experiments [92]. However, we show that considerable complexity can be hidden beyond this apparently simple mean activity.

Figure 4A describes a prokaryotic promoter regulated by a TF $A$, binding cooperatively at two binding sites and competitively with a TF $C$. It also considers the looping of DNA, influencing and influenced by both TFs. All parameters were set to realistic values according to the experimental literature of prokaryotic regulation $[67,79,84,89-91,93,94]$. They are described in detail in the caption of figure 4 and summarized in table S3 of Additional file 1 . In this system, while the mean RNA level as a function of the two TF concentrations reproduce the classical Hill-like four plateaus observed in in vivo experiments [92] (figure 4B1), the normalized variance (figure 4B1) and distribution (figure 4C) reveal a significantly more complex profile carrying much information. Thermodynamic models that only describe the promoter by a free energy $\mathbf{G}^{\mathbf{0}}$ for each state $[79,80]$ are only able to predict the mean of gene expression. Interestingly, in our system, randomizing the activation energies $\mathbf{E}^{\mathbf{0}}$ while keeping the state energies $\mathbf{G}^{\mathbf{0}}$ unchanged does not affect the mean expression at all but has a dramatic impact on the stochastic aspects (figure 4D). This shows that the stochasticity of gene expression, even measured with steady-state metrics, is controlled by and hence can reveal - the dynamics of the promoter. Such an observation not only has strong experimental implications, but also shows that the mean level is not the sole aspect of gene expression that can be regulated and that an elaborate control of the stochastic aspects can also be achieved (cf Discussion).

\section{Dynamic aspects}

The dynamic aspects of promoter activity and in particular the periodic cyclical promoter occupancy and remodeling patters can demonstrate a complex concentration-dependent behavior. We showed earlier that the position of the eigenvalues $\lambda_{i}$ (figure $2 \mathrm{~B}$ ) reflects the dynamics of the whole regulatory structure. Each $\lambda_{i}$ determines the correlation time of aperiodic fluctuations or the frequency and coherence of periodic fluctuations as elementary components of the global dynamics. Since the matrix $\mathbf{M}$ depends on TF concentrations, the position of its eigenvalues change with the concentration of a TF. As illustrated on figure $5 \mathrm{~A}$ with the same system 


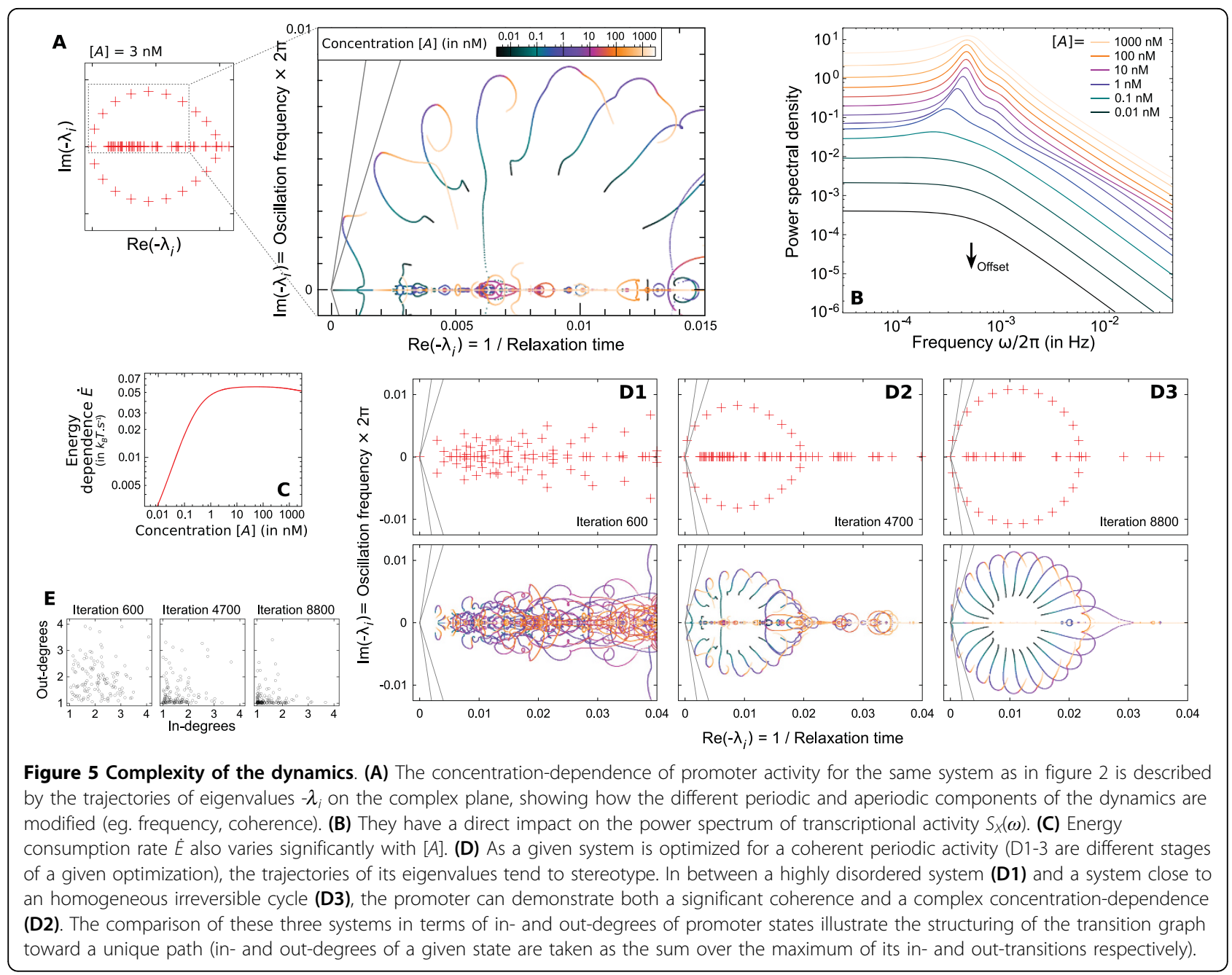

as in figure 2, these eigenvalues can demonstrate nontrivial trajectories. They describe how the temporal characteristics of the periodic loading patterns of molecules change with concentration of TFs and have a direct impact on the resulting transcriptional activity $S_{X}$ $(\omega)$ (figures 5B). Indeed, the basic fact that the concentration of a given TF impacts its association rate not only affects the duration of specific states in the cycle (hence influencing both frequency and coherence), but it also changes the relative probability between the transitions leaving these states. This can have a large variety of effects: influencing the amount of backward reactions in cycles, the probability of outgoing transitions (that can temporarily lead to a local dead-end or to abortion of the cycle), the balance between alternative pathways within a given cycle, and even the commitment of the system between multiple dynamics. Each one of these phenomena has its own effect on the dynamics. In simple ideal systems, these effects could be predicted, but in non-stereotyped system, they can all occur together at different points and with various intensities, resulting in a non-trivial and highly non-linear activity and providing the dynamics with a strong plasticity. Indeed, eigenvalues move along trajectories that can include sharp bends and bifurcations (inducing threshold effects) and with very variable velocity (implying different sensitivity in different concentration ranges). Also, we see that, in this context, energy consumption rate $\dot{E}$ is related but non-directly correlated to the periodic activity of the promoter and that other factors may play a role in their relation (figure $5 \mathrm{C}$ ).

Figures 5D and 5E present three different promoters obtained at different stages of the optimization process used to construct the system of figure 2 . The presented systems range from a very unstructured promoter where states have many in- and out-transitions (figure 5E1) to a stereotyped system close to the homogeneous cycle as indicated by the clear circle on figure 5D3 and the fact that most states have a unique outgoing transition (figure $5 \mathrm{E}$ ). The decreasing complexity observed along the 
optimization process suggest a tradeoff between the coherence of the periodic activity and the flexibility of its regulation. Interestingly, it is at an intermediate level of structuring (figure 5D2), where the system still retains a certain level of disorganization, that a promoter can achieve a significantly coherent dynamics that nonetheless demonstrates a complex concentration-dependent behavior. We argue in Discussion that different features observed on real promoters correspond to this intermediate regime.

\section{Discussion}

\section{The promoter as a central piece in the control of stochasticity}

Stochasticity in gene expression can be advantageous in some situations and harmful in others [15-21]. Therefore, it appears that it should take place at specific times (eg. in response to external factors), on specific genes and with a certain form. We showed how the dynamics of the regulatory structure can induce a large variety of stochastic activities on which a tight control can be exerted. Other mechanisms identified at other stages of the expression process have been shown to influence stochasticity [23,24,27-29,31-34,38,39,41], but none of them provide such a diversity and such a control. Regulation has long been considered as a way to control the mean expression, but it results to be a very powerful and flexible tool for the cell to take advantage of stochasticity by modulating it depending on the context.

Moreover, these capacities require no particular unusual molecular machinery and can be obtained in realistic parameter ranges. They only rely on common features of living systems such as protein-DNA and protein-protein interactions, conformational changes, energy-dependent activity of molecules and, more specific to eukaryotes, modifications of chromatin structure and epigenetic state of histones. Hence, controlling stochasticity is not only possible, but it also appears to be easy to achieve.

\section{Limitations and future work}

Pedraza et al. [41] described the effect on $\sigma_{P}{ }^{2} /\langle P\rangle^{2}$ of considering molecular memories (ie. non-exponential waiting times) on a two-state on/off promoter (represented phenomenologically by bursts of arbitrarily distributed size and waiting time intervals) and on RNA and protein lifetimes. Our work can be viewed as a deep focus on the causes and consequences of the molecular memory that takes place at the promoter. But the fact that indeed RNA and proteins degradations are not first order (the ubiquitin-proteasome system induces nonexponential lifetimes), but also that synthesis are not instantaneous (elongation, splicing, nuclear export ... induce distributed delays) influences the shape of filters in Eq 6 and hence the normalized variance. However, these phenomena only constitute deviations (although potentially strong) from the global tendency that our simple description of these steps can capture.

Protein-protein interactions can occur away from the promoter and can result in the binding of complexes to the promoter (eg. $\varnothing \rightarrow A B$ ) as well as TFs unbinding as complexes (eg. $A B \rightarrow \varnothing$ ). This is taken into account in the general version of the model (cf Additional file 1, $\$ 2)$. In that case, concentrations of complexes are not null and simply imply a larger set of kinetic constants than $\mathbf{k}^{0}$. Still, once the matrix $\mathbf{M}$ is constructed, all the mathematical derivations are strictly identical. Off-promoter TFs associations/dissociations (eg. modeled by a simple reaction network) determine the concentration of free TFs (ie. $[A],[B],[C]$...) and of complexes (ie. $[A B]$, $[B C],[A B C]$...) so that varying the amount of one TF potentially results in variation of concentrations of all TFs and complexes. In particular, if on-promoter and off-promoter interactions are different, this results in the coupling of two different dynamics, leading to additional complexity in eigenvalues trajectories and providing even more flexibility for regulating the dynamic activity of promoters.

This model of gene expression focuses on intrinsic stochasticity of non-autoregulated genes. Going further by considering extrinsic stochasticity and autoregulation could be achieved with various techniques $[23,34,69,70,74,75]$. In the light of the highly non-linear spontaneous behavior we showed, revisiting with the present model properties of signal transmission and stochastic resonance that have been identified with simple models of promoter $[22,28-30,32,38,39,43]$ can already be expected to reveal new properties of gene regulatory structures.

\section{Back to experimentation: what can be expected?}

The in vivo activity of promoters can be explored experimentally as a function of TFs concentrations [73,92]. Typically, Setty et al. [92] measured the mean RNA levels of the lac operon of E. coli as a function of cAMP and IPTG concentrations and interpreted their data in terms of Hill functions. We showed that the steady-state stochastic aspects of expression (that can be obtained by flow cytometry) carry much more information (figure 4). The normalized variance demonstrates a more characteristic profile than the mean level and the distribution itself carries several singularities (varying number/position/size/shape of modes), constituting a real signature of the promoter activity. Indeed, different systems can be obtained showing a large variety of stochastic profiles for a strictly identical mean activity (figure 4D). However, inferring parameters from such measurements appears conceivable only for rather 
simple prokaryotic promoters for which some information is already partially known. Nevertheless, it can reveal the kinetic information that is known to be difficult to obtain [68]. Focusing on mean equilibrium activity, thermodynamic approaches can only infer ratios of pairs of kinetic constants (ie. equilibrium constants) and therefore only state energies $\mathbf{G}^{0}$. On the contrary, our approach can provide the kinetic constants themselves and reveal the energies of the activation barriers $\mathbf{E}^{0}$.

The greater complexity of eukaryotic promoters certainly moderates the success of the previous approach. Nevertheless their singular dynamics promotes the use of time-dependent measures - as obtained by photobleaching [46-48,55], chromatin immunoprecipitation $[49,50,52,66]$ or laser-crosslinking techniques [51] - and ask for the investigation of their concentration-dependent behavior. This would reflect, although partially, trajectories of eigenvalues $\lambda_{i}$ (figure 5A1). For instance, frequency and damping of cyclical occupancy patterns have been observed to change strongly with inducer concentration (R. Métivier, personal communication). Information on the matrix spectrum can also be obtained by timelapse microscopy measurement to follow across time the amount of proteins or transcripts. Giving access to spectral measurements such as $S_{R}(\omega)$ or $S_{P}(\omega)$, this technique was shown to give mechanistic insight into the underlying system $[7,8,12,95]$.

This generic model can thus constitute a unified theoretical framework for all these very different techniques, making them complementary views of the same system.

\section{High mobility, functional redundancy and alternate promoter states}

It appears from our study that a very constrained system that consists in a unique cyclic sequence of events (as [71] and case (ii) in Overview of derivations) results in a simple and stereotyped concentration control ability (eg. figure 5D3). On the other hand, a very random transition graph can demonstrate complex trajectories of eigenvalues but lacks of a coherent periodic activity (eg. figure 5D1). It is when the system has long directed cycles (requiring the presence of energy-dependent transitions) but retains a certain level of disorganization (ie. cycles are not restricted to a unique homogeneous sequence of events but can contain heterogeneities and alternative pathways of variable length and kinetic characteristics) that a complex control of a sensibly coherent periodic activity can occur (eg. figure 5D2). It is worth noting that similar characteristics of molecular interactions have also been proposed to account for a different phenomenon: the generation of a slow population dynamics from rapid molecular kinetics [52]. Interestingly, such properties correspond to what is actually observed experimentally $[48,53,54]$ : Within a slow and coherent periodic dynamics, multiprotein complexes are very unstable due to transient and dynamic interactions of most proteins and can therefore be in a variety of alternate composition. The various proteins species, which recruitment varies along the slow cycle, can have different mobility, therefore inducing kinetic heterogeneities in the slow cycling dynamics. Also, different subcomplexes have been shown to be mutually exclusive but functionally redundant and to form alternatively on the promoter at the same point in the slow cycle, corresponding to stochastic temporary commitments between distinct pathways $[50,53,54]$. This depiction of the dynamics of eukaryotic regulatory structures is likely to result in a complex and flexible concentration dependence. It can even be speculated to have evolved to provide this powerful control mechanism.

\section{The evolutionary cost of the "transcription clock"}

Cyclical patterns of promoter remodeling and occupancy by TFs are often associated to the idea of "transcription clock" [50] for its tendency to provide the dynamics with a certain timing and to synchronize cells in response to external factors. However, as we have shown, imposing periodicities in the dynamics of the regulatory structure requires energy and is limited by the number of possible promoter states. The clear cyclical recruitment of TFs observed in vivo on eukaryotic promoters and its strong coherence indicate a large number of steps and a strong energy-dependence.

Moreover, any deviation from the stereotyped cycle results in a diminution of the coherence of oscillations and makes it suboptimal with respect to energy consumption. This raises an indirect additional cause of energy cost: Achieving a complex concentration-dependent activity (requiring many of such deviations) while keeping a coherent periodic dynamics could represent another evolutionary pressure toward stronger energy consumption and larger regulatory structures.

\section{Conclusion}

In the light of the various capabilities of gene promoters we have demonstrated in this study, it seems that the importance of the single-gene level has to be reconsidered. Indeed, systems biology has set gene networks to the front of the stage, expecting complexity to arise from the interaction of many genes, often considered simple and deterministic. It appears now that single nodes of these networks should be given more attention since their spontaneous stochastic dynamics can be a considerable source of complexity. 
Additional file 1: Supplementary information. This document contains two supplementary figures, an extensive description of the model in different versions and formulations, details of theoretical derivations and all the parameter values of each system presented as example in the main article.

Click here for file

[http://www.biomedcentral.com/content/supplementary/1752-0509-4-2S1.PDF ]

\section{Acknowledgements}

The authors want to thank Raphaël Métivier for helpful discussions and comments on this work, David Parsons for critical reading of the manuscript, as well as Daniel Kahn, Jean-Jacques Kupiec and the members of the Biologie des Systèmes et Modélisation Cellulaire (BSMC) group for discussions. This work was funded by the Rhône-Alpes region (ISLE cluster), the Rhône-Alpes complex systems institute (IXXI) and the french national network of complex systems (RNSC).

\section{Author details}

'Université de Lyon, Université Lyon 1, Centre de Génétique Moléculaire et Cellulaire (CGMC), CNRS UMR5534, F-69622 Lyon, France. 'Université de Lyon, INSA-Lyon, Laboratoire d'InfoRmatique en Image et Systemes d'information (LIRIS), CNRS UMR5205, F-69621 Lyon, France. ${ }^{3}$ Rhône-Alpes Complex Systems Institute (IXXI), F-69007 Lyon, France.

\section{Authors' contributions}

$A C, O G$ and $G B$ designed the study. AC performed the theoretical derivations of the model and conceived/analysed the different systems presented as examples. AC, OG and GB interpreted the results and wrote the paper. All authors read and approved the final manuscript.

Received: 2 August 2009

Accepted: 8 January 2010 Published: 8 January 2010

\section{References}

1. Elowitz M, Levine A, Siggia E, Swain P: Stochastic gene expression in a single cell. Science 2002, 297:1183-1186.

2. Blake WJ, Kærn M, Cantor CR, Collins JJ: Noise in eukaryotic gene expression. Nature 2003, 422:633-7.

3. Raser J, O'Shea E: Control of stochasticity in eukaryotic gene expression. Science 2004, 304:1811-1814.

4. Golding I, Paulsson J, Zawilski S, Cox E: Real-time kinetics of gene activity in individual bacteria. Cell 2005, 123:1025-1036.

5. Becskei A, Kaufmann B, van Oudenaarden A: Contributions of low molecule number and chromosomal positioning to stochastic gene expression. Nat Genet 2005, 37:937-944.

6. Raj A, Peskin C, Tranchina D, Vargas D, Tyagi S: Stochastic mRNA synthesis in mammalian cells. PLOS Biol 2006, 4:e309.

7. Sigal A, Milo R, Cohen A, Geva-Zatorsky N, Klein Y, Liron Y, Rosenfeld N, Danon T, Perzov N, Alon U: Variability and memory of protein levels in human cells. Nature 2006, 444:643-646.

8. Austin D, Allen M, McCollum J, Dar R, Wilgus J, Sayler G, Samatova N, Cox C, Simpson M: Gene network shaping of inherent noise spectra. Nature 2006, 439:608-611.

9. Volfson D, Marciniak J, Blake W, Ostroff N, Tsimring L, Hasty J: Origins of extrinsic variability in eukaryotic gene expression. Nature 2006, 439:861-864.

10. Neildez-Nguyen T, Parisot A, Vignal C, Rameau P, Stockholm D, Picot J, Allo V, Le Bec C, Laplace C, Paldi A: Epigenetic gene expression noise and phenotypic diversification of clonal cell populations. Differentiation 2007, 76:33-40.

11. Ansel J, Bottin H, Rodriguez-Beltran C, Damon C, Nagarajan M, Fehrmann S, François J, Yvert G: Cell-to-cell stochastic variation in gene expression is a complex genetic trait. PLoS Genet 2008, 4:e1000049.

12. Dunlop M, Cox R, Levine J, Murray R, Elowitz M: Regulatory activity revealed by dynamic correlations in gene expression noise. Nat Genet 2008, 40:1493-1498.
13. Maheshri N, O'Shea E: Living with noisy genes: how cells function reliably with inherent variability in gene expression. Annu Rev Biophys Biomol Struct 2007, 36:413-434.

14. Kaufmann B, van Oudenaarden A: Stochastic gene expression: from single molecules to the proteome. Curr Opin Genet Dev 2007, 17:107-112.

15. Raj A, van Oudenaarden A: Nature, nurture, or chance: stochastic gene expression and its consequences. Cell 2008, 135(2):216-26.

16. Martinez Arias A, Hayward P: Filtering transcriptional noise during development: concepts and mechanisms. Nat Rev Genet 2006, 7:34-44.

17. Süel G, Kulkarni R, Dworkin J, Garcia-Ojalvo J, Elowitz M: Tunability and noise dependence in differentiation dynamics. Science 2007, 315:1716-1719.

18. Chang H, Hemberg M, Barahona M, Ingber D, Huang S: Transcriptomewide noise controls lineage choice in mammalian progenitor cells. Nature 2008, 453:544-547.

19. Samoilov M, Price G, Arkin A: From fluctuations to phenotypes: the physiology of noise. SCi STKE 2006, 2006:re17.

20. Veening J, Smits W, Kuipers O: Bistability, Epigenetics, and Bet-Hedging in Bacteria. Annu Rev Microbiol 2008, 62:193-210.

21. Kupiec JJ: A Darwinian theory for the origin of cellular differentiation. Mol Gen Genet 1997, 255:201-208.

22. Swain PS, Elowitz MB, Siggia ED: Intrinsic and extrinsic contributions to stochasticity in gene expression. Proc Natl Acad Sci USA 2002, 99(20):12795-800.

23. Paulsson J: Models of stochastic gene expression. Phys Life Rev 2005, 2(2):157-75.

24. Rigney DR, Schieve WC: Stochastic model of linear, continuous protein synthesis in bacterial populations. J Theor Biol 1977, 69:761-766.

25. Ko MS: A stochastic model for gene induction. J Theor Biol 1991 153:181-194.

26. Peccoud J, Ycart B: Markovian Modeling of Gene-Product Synthesis. Theoretical Population Biology 1995, 48(2):222-234.

27. Kepler TB, Elston TC: Stochasticity in Transcriptional Regulation: Origins, Consequences, and Mathematical Representations. Biophys J 2001, 81(6):3116-36.

28. Thattai $M$, van Oudenaarden $A$ : Intrinsic noise in gene regulatory networks. Proc Natl Acad Sci USA 2001, 98(15):8614-9.

29. Simpson M, Cox C, Sayler G: Frequency domain analysis of noise in autoregulated gene circuits. Proc Natl Acad Sci USA 2003, 100:4551-4556.

30. Simpson M, Cox C, Sayler G: Frequency domain chemical Langevin analysis of stochasticity in gene transcriptional regulation. $J$ Theor Biol 2004, 229:383-394.

31. Swain P: Efficient attenuation of stochasticity in gene expression through post-transcriptional control. J Mol Biol 2004, 344:965-976.

32. Paulsson J: Summing up the noise in gene networks. Nature 2004, 427:415-8.

33. van Zon J, Morelli M, Tanase-Nicola S, ten Wolde P: Diffusion of transcription factors can drastically enhance the noise in gene expression. Biophys J 2006, 91:4350-4367.

34. Cox CD, McCollum JM, Austin DW, Allen MS, Dar RD, Simpson ML: Frequency domain analysis of noise in simple gene circuits. Chaos 2006, 16:026102.

35. Lipniacki T, Paszek P, Marciniak-Czochra A, Brasier A, Kimmel M: Transcriptional stochasticity in gene expression. J Theor Biol 2006, 238:348-367.

36. Paszek P: Modeling stochasticity in gene regulation: characterization in the terms of the underlying distribution function. Bull Math Biol 2007, 69:1567-1601.

37. Innocentini $\mathrm{G}$, Hornos J: Modeling stochastic gene expression under repression. J Math Biol 2007, 55:413-431.

38. Tao $Y$, Zheng $X$, Sun $Y$ : Effect of feedback regulation on stochastic gene expression. J Theor Biol 2007, 247:827-836.

39. Hornung $G$, Barkai N: Noise propagation and signaling sensitivity in biological networks: a role for positive feedback. PLoS Comput Biol 2008, 4:e8.

40. Shahrezaei V, Swain PS: Analytical distributions for stochastic gene expression. Proc Natl Acad Sci USA 2008, 105(45):17256-61.

41. Pedraza J, Paulsson J: Effects of molecular memory and bursting on fluctuations in gene expression. Science 2008, 319:339-343. 
42. Sánchez A, Kondev J: Transcriptional control of noise in gene expression. Proc Natl Acad Sci USA 2008, 105:5081-5086.

43. Warmflash A, Dinner A: Signatures of combinatorial regulation in intrinsic biological noise. Proc Natl Acad Sci USA 2008, 105:17262-17267.

44. Mitarai N, Dodd IB, Crooks MT, Sneppen K: The generation of promotermediated transcriptional noise in bacteria. PLoS Comput Biol 2008, 4: e1000109.

45. McNally J, Müller W, Walker D, Wolford R, Hager G: The glucocorticoid receptor: rapid exchange with regulatory sites in living cells. Science 2000, 287:1262-1265.

46. Becker M, Baumann C, John S, Walker D, Vigneron M, McNally J, Hager G: Dynamic behavior of transcription factors on a natural promoter in living cells. EMBO Rep 2002, 3:1188-1194.

47. Phair R, Scaffdi P, Elbi C, Vecerová J, Dey A, Ozato K, Brown D, Hager G, Bustin M, Misteli T: Global nature of dynamic protein-chromatin interactions in vivo: three-dimensional genome scanning and dynamic interaction networks of chromatin proteins. Mol Cell Biol 2004, 24:6393-6402.

48. Karpova TS, Kim MJ, Spriet C, Nalley K, Stasevich TJ, Kherrouche Z, Heliot L, McNally JG: Concurrent fast and slow cycling of a transcriptional activator at an endogenous promoter. Science 2008, 319:466-469.

49. Shang Y, Hu X, DiRenzo J, Lazar MA, Brown M: Cofactor dynamics and sufficiency in estrogen receptor-regulated transcription. Cell 2000, 103:843-852.

50. Métivier R, Penot G, Hubner M, Reid G, Brand H, Kos M, Gannon F: Estrogen receptor-alpha directs ordered, cyclical, and combinatorial recruitment of cofactors on a natural target promoter. Cell 2003, 115:751-763.

51. Nagaich A, Walker D, Wolford R, Hager G: Rapid periodic binding and displacement of the glucocorticoid receptor during chromatin remodeling. Mol Cell 2004, 14:163-174.

52. Degenhardt T, Rybakova KN, Tomaszewska A, Moné MJ, Westerhoff HV, Bruggeman FJ, Carlberg C: Population-level transcription cycles derive from stochastic timing of single-cell transcription. Cell 2009, 138:489-501.

53. Métivier R, Reid G, Gannon F: Transcription in four dimensions: nuclear receptor-directed initiation of gene expression. EMBO Rep 2006, 7:161-167.

54. Hager G, Elbi C, Johnson T, Voss T, Nagaich A, Schiltz R, Qiu Y, John S: Chromatin dynamics and the evolution of alternate promoter states. Chromosome Res 2006, 14:107-116.

55. Misteli T: Beyond the sequence: cellular organization of genome function. Cell 2007, 128:787-800.

56. Browning D, Busby S: The regulation of bacterial transcription initiation. Nat Rev Microbiol 2004, 2:57-65.

57. Dodd I, Shearwin K, Perkins A, Burr T, Hochschild A, Egan J: Cooperativity in long-range gene regulation by the lambda $\mathrm{Cl}$ repressor. Genes Dev 2004, 18:344-354.

58. Adams C, Workman J: Binding of disparate transcriptional activators to nucleosomal DNA is inherently cooperative. Mol Cell Biol 1995, 15:1405-1421.

59. Agresti A, Scaffidi P, Riva A, Caiolfa V, Bianchi M: GR and HMGB1 interact only within chromatin and influence each other's residence time. Mol Cell 2005, 18:109-121.

60. Mellor J: Dynamic nucleosomes and gene transcription. Trends Genet 2006, 22:320-329.

61. Li B, Carey M, Workman J: The role of chromatin during transcription. Cell 2007, 128:707-719.

62. Jenuwein T, Allis C: Translating the histone code. Science 2001, 293:1074-1080.

63. Verdone L, Caserta M, Di Mauro E: Role of histone acetylation in the control of gene expression. Biochem Cell Biol 2005, 83:344-353.

64. Wang Z, Zang C, Rosenfeld J, Schones D, Barski A, Cuddapah S, Cui K, Roh T, Peng W, Zhang M, Zhao K: Combinatorial patterns of histone acetylations and methylations in the human genome. Nat Genet 2008, 40:897-903.

65. Voss TC, Schiltz RL, Sung MH, Johnson TA, John S, Hager GL: Combinatorial probabilistic chromatin interactions produce transcriptional heterogeneity. J Cell Sci 2009, 122:345-356.

66. Métivier R, Gallais R, Tiffoche C, Le Péron C, Jurkowska R, Carmouche R, Ibberson D, Barath P, Demay F, Reid G, Benes V, Jeltsch A, Gannon F,
Salbert G: Cyclical DNA methylation of a transcriptionally active promoter. Nature 2008, 452:45-50.

67. Vilar J, Saiz L: DNA looping in gene regulation: from the assembly of macromolecular complexes to the control of transcriptional noise. Curr Opin Genet Dev 2005, 15:136-144.

68. Saiz L, Vilar J: Stochastic dynamics of macromolecular-assembly networks. Mol Syst Biol 2006, 2:2006 0024

69. Warren $\mathrm{P}$, Tanase-Nicola S, ten Wolde P: Exact results for noise power spectra in linear biochemical reaction networks. J Chem Phys 2006, 125:144904

70. Lestas I, Paulsson J, Ross N, Vinnicombe G: Noise in Gene Regulatory Networks. IEEE Transactions 2008, 53:189-200.

71. Lemaire V, Lee C, Lei J, Métivier R, Glass L: Sequential recruitment and combinatorial assembling of multiprotein complexes in transcriptional activation. Phys Rev Lett 2006, 96:198102.

72. Benecke A: Chromatin code, local non-equilibrium dynamics, and the emergence of transcription regulatory programs. Eur Phys J E Soft Matter 2006, 19:353-366.

73. Kim HD, O'Shea EK: A quantitative model of transcription factor-activated gene expression. Nat Struct Mol Biol 2008, 15(11):1192-8.

74. Goutsias J: A hidden Markov model for transcriptional regulation in single cells. IEEE/ACM Trans Comput Biol Bioinform 2006, 3:57-71.

75. van Kampen N: Stochastic processes in physics and chemistry New York: North-Holland, 32007.

76. Ribeiro AS, Lloyd-Price J: SGN Sim, a stochastic genetic networks simulator. Bioinformatics 2007, 23:777-779.

77. Chabrier-Rivier N, Fages F, Soliman S: The Biochemical Abstract Machine BIOCHAM. Computational Methods in Systems Biology Springer 2005, 3082:172-91.

78. Kuttler C: Simulating Bacterial Transcription and Translation in a Stochastic $\pi$ Calculus. Transactions on Computational Systems Biology VI Springer 2006, 4220:113-49.

79. Ackers $G$, Johnson A, Shea M: Quantitative model for gene regulation by lambda phage repressor. Proc Natl Acad Sci USA 1982, 79:1129-1133.

80. Bintu L, Buchler N, Garcia H, Gerland U, Hwa T, Kondev J, Phillips R: Transcriptional regulation by the numbers: models. Curr Opin Genet Dev 2005, 15:116-124.

81. Li G, Levitus M, Bustamante C, Widom J: Rapid spontaneous accessibility of nucleosomal DNA. Nat Struct Mol Biol 2005, 12:46-53.

82. Tomschik M, van Holde K, Zlatanova J: Nucleosome dynamics as studied by single-pair fluorescence resonance energy transfer: a reevaluation. $J$ Fluoresc 2009, 19:53-62.

83. Lee TI, Young RA: Regulation of gene expression by TBP-associated proteins. Genes Dev 1998, 12:1398-1408.

84. Lu P, Vogel C, Wang R, Yao X, Marcotte EM: Absolute protein expression profiling estimates the relative contributions of transcriptional and translational regulation. Nat Biotechnol 2007, 25:117-124.

85. Wang Y, Liu CL, Storey JD, Tibshirani RJ, Herschlag D, Brown PO: Precision and functional specificity in mRNA decay. Proc Natl Acad Sci USA 2002, 99:5860-5865.

86. Belle A, Tanay A, Bitincka L, Shamir R, O'Shea EK: Quantification of protein half-lives in the budding yeast proteome. Proc Natl Acad Sci USA 2006, 103:13004-13009.

87. Hearon JZ: The kinetics of linear systems with special reference to periodic reactions. Bull Math Biol 1953, 15:121-141.

88. Berg JM, Tymoczko JL, Stryer L: Biochemistry New York: W. H. Freeman and Company, 52002.

89. Bintu L, Buchler N, Garcia H, Gerland U, Hwa T, Kondev J, Kuhlman T, Phillips R: Transcriptional regulation by the numbers: applications. Curr Opin Genet Dev 2005, 15:125-135.

90. Wang Y, Guo L, Golding I, Cox EC, Ong NP: Quantitative transcription factor binding kinetics at the single-molecule level. Biophys J 2009, 96:609-620.

91. Bernstein JA, Khodursky AB, Lin PH, Lin-Chao S, Cohen SN: Global analysis of mRNA decay and abundance in Escherichia coli at single-gene resolution using two-color fluorescent DNA microarrays. Proc Natl Acad Sci USA 2002, 99:9697-9702.

92. Setty $Y$, Mayo A, Surette M, Alon U: Detailed map of a cis-regulatory input function. Proc Natl Acad Sci USA 2003, 100:7702-7707. 
93. Saiz L, Vilar JM: DNA looping: the consequences and its control. Curr Opin Struct Biol 2006, 16:344-350.

94. Saiz L, Rubi JM, Vilar JM: Inferring the in vivo looping properties of DNA. Proc Natl Acad Sci USA 2005, 102:17642-17645.

95. Cox CD, McCollum JM, Allen MS, Dar RD, Simpson ML: Using noise to probe and characterize gene circuits. Proc Natl Acad Sci USA 2008, 105:10809-10814.

doi:10.1186/1752-0509-4-2

Cite this article as: Coulon et al:: On the spontaneous stochastic

dynamics of a single gene: complexity of the molecular interplay at the promoter. BMC Systems Biology 2010 4:2.

Submit your next manuscript to BioMed Central and take full advantage of:

- Convenient online submission

- Thorough peer review

- No space constraints or color figure charges

- Immediate publication on acceptance

- Inclusion in PubMed, CAS, Scopus and Google Scholar

- Research which is freely available for redistribution

Submit your manuscript at www.biomedcentral.com/submit 